\title{
Essais
}

ESSAIS

Revue interdisciplinaire d'Humanités

5 | 2014

Médias et élites

\section{La presse face aux élites politiques et économiques : le cas du Matin (1884-1944)}

Dominique Pinsolle

\section{OpenEdition}

1 Journals

Édition électronique

URL : http://journals.openedition.org/essais/8163

DOI : 10.4000/essais. 8163

ISSN : 2276-0970

Éditeur

École doctorale Montaigne Humanités

Édition imprimée

Date de publication : 15 juillet 2014

Pagination : 56-66

ISBN : 978-2-9544269-3-8

ISSN : 2417-4211

Référence électronique

Dominique Pinsolle, "La presse face aux élites politiques et économiques : le cas du Matin

(1884-1944) », Essais [En ligne], 5 | 2014, mis en ligne le 13 avril 2021, consulté le 15 avril 2021. URL:

http://journals.openedition.org/essais/8163; DOI : https://doi.org/10.4000/essais.8163 


\section{La presse face aux élites politiques et économiques : le cas du Matin (1884-1944)}

\section{Dominique Pinsolle}

Les journalistes sont une des incarnations des nouvelles professions intellectuelles qui montent en puissance au cours du XIX siècle $^{1}$. La frange la plus reconnue et la plus influente d'entre eux fait assurément partie des élites intellectuelles, entendues ici comme la fraction des élites culturelles ${ }^{2}$ dont la position dominante repose avant tout sur un capital symbolique accumulé grâce à ses interventions dans l'espace public ${ }^{3}$. Le journaliste occupe effectivement une place centrale dans le modèle démocratique de la III ${ }^{e}$ République, dans la mesure où le citoyen a besoin d'être informé pour être " éclairé ». Cependant, par sa nature même, la presse est condamnée à être sans cesse soumise à des pressions provenant du monde politique ou des milieux d'affaires : le contrôle de l'information est un levier d'influence, qui suscite nombre de convoitises ${ }^{4}$. De ce fait, les journalistes, tout en faisant partie, pour certains d'entre eux, d'une nouvelle élite intellectuelle, entretiennent des rapports ambigus avec les élites politiques et économiques. D'un côté, la presse entend et prétend exercer un contre-pouvoir (consacré par la loi de 1881 qui l'affranchit de la tutelle étatique), mais, de l'autre, elle fait constamment l'objet de tentatives d'instrumentalisation par des hommes politiques ou des brasseurs d'affaires peu scrupuleux, qui parviennent souvent à leurs fins. Dans ces conditions, les journaux d'information s'apparentent parfois davantage à des auxiliaires du pouvoir qu’à un " quatrième

1 Delporte Christian, Les journalistes en France, 1880-1950. Naissance et construction d'une profession, Paris, Seuil, 1999, 450 p.

2 Nous nous appuyons ici sur les définitions développées par Jean-François Sirinelli, «Les élites culturelles ", in Rioux Jean-Pierre et Sirinelli Jean-François (éd.), Pour une histoire culturelle, Paris, Seuil, 1997, 455 p. et Christophe Charle, Les élites de la République, 1880-1900, Paris, Fayard, 2006, 613 p., p. 23-24.

3 Nous reprenons ici le concept d' "espace public » développé par Jürgen Habermas in L'Espace public. Archéologie de la publicité comme dimension constitutive de la société bourgeoise, Paris, Payot, 1978 (1962).

4 Champagne Patrick, "La double dépendance. Quelques remarques sur les rapports entre les champs politique, économique et journalistique ", Hermès, 17-18, 1995, p. 215-229. 
pouvoir ", et la réalité de leurs pratiques en vient, dans de nombreuses circonstances, à contredire l'idéal d'une presse libre au service de la vérité défiant les puissants. Cela signifie-t-il qu'en étant intégrés au monde des élites, les journalistes les plus influents sont passés au service des élites? Sans prétendre apporter une répondre définitive et globale à cette question, il est possible d'étudier les mécanismes par lesquels la presse peut être instrumentalisée par le monde politique (particulièrement les gouvernants ou les hommes politiques les plus influents) et les milieux d'affaires (composés des individus occupant les positions dominantes dans le champ économique ${ }^{5}$ ). Nous nous concentrerons ici sur l'exemple du journal Le Matin, fondé en 1884 et disparu en 1944, qui est un des titres français les plus importants durant la première moitié du $\mathrm{XX}^{\mathrm{e}}$ siècle. Ce quotidien entretient un rapport particulièrement ambigu aux élites et révèle, par ses excès, la complexité des relations entre le pouvoir politique, le monde des affaires et la presse de la III République. D'une part, par ses campagnes retentissantes et son ton tapageur, il se présente comme un organe au service du peuple contre des élites politiques impuissantes et corrompues. D'autre part, il fait figure de quotidien lui-même corrompu, aux mains d'un affairiste sans scrupules prêt à mettre son journal au service des puissants du moment, qu'ils soient gouvernants ou hommes d'affaires. Enfin, les relations qu'il entretient avec le monde politique sont révélatrices à la fois de la puissance de son patron, mais aussi de sa fragilité face aux élites traditionnelles.

\section{Le Matin : l'incarnation de la nouvelle élite journalistique méprisée par les élites traditionnelles}

Lors de son lancement, en 1884, Le Matin incarne la nouvelle presse " à l'américaine " qui tente de s'implanter en France, mais aussi l'avènement d'une nouvelle élite journalistique entretenant des rapports ambigus avec les élites traditionnelles, qu'elles soient politiques ou économiques. La création du Matin par des hommes d'affaires américains est à replacer dans le contexte de la professionnalisation du journalisme et de la constitution d'un champ journalistique marqué par le développement d'une presse marchande s'opposant à la presse de tradition politique et littéraire. En effet, à la fin du XIX ${ }^{\mathrm{e}}$ siècle, la figure du reporter occulte progressivement celle de l'écrivain ou du militant, et le journalisme cesse d'être une activité annexe ou secondaire pour devenir l'activité principale des journalistes, censés se soumettre à un certain nombre de prin-

5 Charle Christophe, «Les milieux d'affaires dans la structure de la classe dominante vers 1900 », Actes de la Recherche en Sciences Sociales, vol. 20-21, mars-avril 1978, p. 83-96 ; Jeanneney JeanNoël, L'argent caché. Milieux d'affaires et pouvoirs politiques dans la France du XX' siècle, Paris, Fayard, Points Histoire, 1984, 306 p.

6 Pinsolle Dominique, Le Matin (1884-1944). Une presse d'argent et de chantage, préface de Christian Delporte, Rennes, Presses universitaires de Rennes, coll. Histoire, 2012, 354 p. 
cipes déontologiques ${ }^{7}$. Le Matin est parfaitement représentatif de l'émergence d'un nouveau type de journalisme fondé sur la notion d' " objectivité ", mettant en avant la rigueur professionnelle et l'attachement aux faits, afin de se distinguer de l'engagement des feuilles militantes et des commentaires subjectifs de la presse littéraire ${ }^{8}$. Alors que la presse n'était historiquement qu'une création des élites traditionnelles, les nouveaux journaux dont Le Matin se veut l'avant-garde sont animés par des professionnels venus de couches sociales moins élevées que celles dont étaient issus jusque-là les publicistes. Leur rapport plus distant au pouvoir, au moins dans le discours, n'est certainement pas étranger au fait que ces nouveaux journalistes appartiennent pour la plupart aux classes moyennes supérieures (artisans et commerçants, enseignants, acteurs, littérateurs, publicistes, hommes de science...), et que leur ascension a entraîné l'éviction des " catégories sociales les plus élevées et les plus traditionalistes " (vieilles familles aristocratiques ou bourgeoises, industriels, banquiers, armateurs, négociants, officiers, magistrats... $)^{9}$. Précisons cependant que, si le journalisme se démocratise (comme l'illustre Bel Ami de Maupassant, en 1884), il reste un milieu où les catégories sociales les plus modestes (ouvriers, paysans, familles pauvres...) sont très peu représentées ${ }^{10}$.

Le champ journalistique est donc en pleine mutation lorsqu'est lancé Le Matin, en 1884. Ce dernier, qui incarne une nouvelle conception de l'information ainsi qu'un nouveau rapport de la presse aux élites traditionnelles, cristallise dès sa parution les débats autour de l' "américanisation » de la presse, et, plus largement, du déclin culturel et intellectuel du journalisme français. Les nouveaux journalistes sont effectivement vus avec beaucoup de mépris par les élites traditionnelles. Émile Zola estime, dès 1877, que la presse a perdu sa "dignitié ». Vingt ans plus tard, Henry Bérenger publie une enquête devenue célèbre dans la Revue bleue, pour dénoncer l'avilissement de la presse devenue un commerce, l'abandon de la noblesse des lettres et de la réflexion au profit de l'argent ${ }^{11}$. C'est Anatole Leroy-Beaulieu qui, en 1903, exprime le mieux cette aversion du monde des lettres envers une nouvelle presse accusée d'avoir brisé le lien qui rattachait jusque-là les journaux à l'élite :

La presse s'est abaissée et s'est corrompue en se vulgarisant. Autrefois elle était rédigée par une élite pour une élite. Aujourd'hui, s'il reste encore une presse d'élite sérieuse, digne de sa haute mission, elle est submergée par une presse nouvelle, moins soucieuse des idées que des intérêts, moins curieuse, d'ins-

7 Palmer Michael B., Des petits journaux aux grandes agences. Naissance du journalisme moderne, Paris, Aubier, 1983, 347 p.

8 Ferenczi Thomas, Linvention du journalisme en France. Naissance de la presse moderne à la fin du XIXe siècle, Paris, Payot, 1996, 276 p.

9 Martin Marc, Médias et journalistes de la République, Paris, Odile Jacob, 1997, 494 p., p. 61.

10 Ibid., p. 125.

11 Christian Delporte, Les journalistes en France, op. cit., p. 131-137. 
truire que d'amuser, jalouse avant tout de plaire, et résignée, pour plaire, à flatter les préjugés, les vices, les passions, les ignorances des lecteurs ${ }^{12}$.

Ce mépris exprimé par les élites traditionnelles pousse les journalistes à mettre en avant les vertus dont ils s'estiment parés et à développer une sorte de mythologie professionnelle censée légitimer leur travail : les journalistes sont les garants de la démocratie, les défenseurs désintéressés du peuple et de la justice, les serviteurs acharnés de l'intérêt public... Le Matin se fait une spécialité de ce type de profession de foi, comme le 23 août 1903, lorsqu'il s'attribue un rôle déterminant dans la condamnation de l'escroc Thérèse Humbert :

[M]algré toutes les calomnies qu'on débite sur elle, la Presse a du bon. C'est encore elle, quoi qu'on en dise au Palais et ailleurs, qui demeure la gardienne vigilante de la vérité, parce qu'elle s'inspire de cette conscience publique qui n’admet ni les silences coupables ni les dégradantes capitulations. [...] Décriée, calomniée, vilipendée, la Presse sincère, celle qui n’a souci que du bien public, monsieur l'avocat général, poursuit son œuvre sans s'occuper des médisants.

Cependant, force est de constater que la légitimation de cette nouvelle catégorie professionnelle ne va pas de soi et qu'elle reste l'objet d'une tension perpétuelle, qui refait surface à chaque fois que l'indépendance des journalistes est remise en cause ou que la logique commerciale à laquelle les médias sont soumis est dénoncée.

\section{L'imbrication entre la presse et les élites économiques}

Cette tension est directement liée au fait que la presse, dans la forme nouvelle qu'elle prend dans la seconde moitié du XIX ${ }^{\mathrm{e}}$ siècle, est un produit du monde des affaires. Développement de la presse et essor de la bourse sont parallèles, et la " financiarisation » des journaux est un processus qui accompagne l'avènement d'un nouveau modèle journalistique en France à partir des années 1830, fondé sur le financement par la publicité et la constitution des entreprises de presse sur le modèle de la société anonyme dont les actions s'échangent à la bourse ${ }^{13}$. Les journaux attirent ainsi des hommes d'affaires soucieux d'investir dans un secteur en pleine expansion et susceptible de leur procurer d'importants dividendes. Cette " financiarisation " impose par la même occasion une nouvelle conception de la presse : le journal doit désormais être un produit rentable, attirant un lectorat nombreux qui puisse être lui-même "revendu " aux annonceurs. La formule connaît un formidable succès : la presse est un des principaux secteurs d'investissement à la Bourse ${ }^{14}$.

12 Cité dans ibid., p. 132.

13 Pradié Christian, La presse, le capitalisme et le lecteur. Contribution à l'histoire économique d'une industrie culturelle, 2 vol., Thèse en sciences de l'information et de la communication, sous la direction de Bernard Miège, Grenoble 3, 1994, 814 p. 
Mais ce processus entraîne la soumission des journaux aux milieux d'affaires, et donc à une certaine catégorie d'élites économiques dont l'influence, contrairement à celle des gouvernants, n’est pas remise en question par la loi de 1881 . Le Législateur ne dissocie alors pas liberté politique et liberté économique. À vrai dire, les questions touchant à la structure financière et commerciale des journaux restent marginales et la question de la soumission des journaux au pouvoir de l'argent et au monde des affaires semble " hors de propos ${ }^{15}$ ". Les perspectives de profits attirent alors d'autant plus les investisseurs et les spéculateurs ; en 1881, 73 titres sont cotés, dont Le Siècle, Le Figaro, Le Petit Journal, Le Temps, Le XIX $X^{e}$ Siècle, Le Petit XIX $X^{e}$ Siècle et, plus généralement, la plupart des journaux républicains (Le Petit Républicain, La Lanterne, La Paix, Le Petit Parisien, Le Rappel, La Marseillaise, Le Nouveau Journal, Le Temps, etc.), une bonne part des titres monarchistes (Le Gaulois, La France Nouvelle, L'Estafette, etc.), plusieurs journaux locaux et une partie de la presse spécialisée ${ }^{16}$. Comme l'explique Jules Simon dans Le Matin du 12 août 1884 : "Aujourd'hui, ce ne sont plus les partis qui créent les journaux, ce sont les banquiers. Ils les créent pour lancer une affaire. Ils choisissent la politique qu'ils croient la plus propre à leur fournir des actionnaires ${ }^{17}$ ".

L'imbrication entre presse et monde des affaires, qui contredit le discours d'indépendance vertueuse tenu par la profession elle-même, se constate également sur le plan spatial. La première société éditrice du Morning News (quotidien parisien de langue anglaise qui donne plus tard naissance au Matin), constituée le 23 octobre 1883, a son siège au 15, rue Daunou, dans le deuxième arrondissement (près de l'opéra Garnier), soit au cour de l'espace parisien de la presse qui s'est constitué autour de la poste centrale et du palais Brongniart. La seconde société (créée l'année suivante) n'est pas loin : son siège se trouve au 25, rue d'Argenteuil (dans le premier arrondissement). En 1899 , le journal déménage au 6 , boulevard Poissonnière : désormais, c'est sur les grands boulevards que, d'est en ouest, "s'égrènent les sièges des quotidiens, du Temps boulevard des Italiens, au Matin, boulevard Poissonnière" ${ }^{18}$ ". C'est aussi dans ce quartier que " les théâtres, la Bourse, les banques, les grands magasins, les gares de chemins de fer et bientôt les principaux centraux téléphoniques $»$ ont leurs sièges ${ }^{19}$.

Le premier patron du Matin lui-même, Alfred Edwards, fils d'un médecin reconverti dans les opérations financières douteuses, est, certes, un journaliste, mais aussi un affairiste sans scrupules qui n’hésite pas à utiliser son journal pour extorquer de l'argent à la Compagnie de Panama (entre 1883 et 1888), ou

15 Delporte Christian, Les journalistes en France, op. cit., p. 29.

16 Pradié Christian, La presse, le capitalisme et le lecteur, vol. 1, op. cit., p. 73.

17 Cité in Palmer Michael B., Des petits journaux aux grandes agences, op. cit., p. 97.

18 Delporte Christian, Les journalistes en France, op. cit., p. 111.

19 Eveno Patrick, L'argent de la presse française des années 1820 à nos jours, Paris, CTHS, 237 p., p. 48. 
pour lancer une opération de chantage contre le directeur du Crédit foncier (en $1890)^{20}$. Son successeur, Maurice Bunau-Varilla, est encore pire. D'abord petit spéculateur à la Bourse, il s'enrichit avec son frère en escroquant la Compagnie de Panama à la fin des années 1880, avant d'investir sa fortune en partie frauduleuse dans Le Matin. Le secteur de la presse est alors d'autant plus attractif que les investissements dans le secteur des travaux publics et de la construction des logements, qui ont connu un véritable «boom » dans les années 1878-1883 avant de s'effondrer jusqu'en 1886, sont dans une période de stagnation, car la France est en situation de suréquipement ${ }^{21}$. La presse peut donc intéresser des entrepreneurs de travaux publics soucieux de défendre leurs intérêts et d'investir dans un secteur en pleine expansion : le secteur presse-édition, avec un taux de croissance de 4,5\% entre 1896 et 1913, fait alors partie du "peloton de tête " des "industries du XX e siècle "; durant la même période, le taux de croissance du secteur du bâtiment et des travaux publics n'est que de $2,1 \%{ }^{22}$. Bunau-Varilla n'est pas le seul à percevoir l'intérêt d'investir dans la presse : Eugène Letellier qui, lui aussi, a participé aux chantiers du canal de Panama, achète avec son frère Léon Le Journal, concurrent du Matin; quant au Temps, il est aux mains d'Adrien Hébrard qui, en tant qu'entrepreneur de travaux publics, a également été lié aux affaires de Gustave Eiffel à Panama.

Maurice Bunau-Varilla, qui reste pendant 40 ans à la tête de la "Maison rouge " (qui doit son nom à la couleur de ses immeubles, boulevard Poissonnière), doit en grande partie sa carrière à son frère Philippe, ingénieur resté célèbre pour le rôle qu'il a joué dans la construction du canal de Panama. Ce dernier fait partie de ces hommes nouveaux qui parviennent, grâce à leur formation, à occuper des positions dominantes dans le champ économique à la fin du XIX ${ }^{\mathrm{e}}$ siècle. Comme le souligne Christophe Charle, la profession d'ingénieur permet alors à des individus aux origines sociales moyennes d'accéder aux affaires et, plus généralement, d'exercer des responsabilités de direction au plus haut niveau ${ }^{23}$. Philippe Bunau-Varilla est ainsi un parfait représentant de ces hommes qui, ne faisant pas partie des héritiers, échappent à leur destin social grâce à leurs titres ou « leur apprentissage sur le tas " et à leurs investissements dans les secteurs nouveaux comme les grands travaux à l'étranger ${ }^{24}$. Maurice, quant à lui, ne fait pas de grandes études ${ }^{25}$ et n'accède

20 Pinsolle Dominique, «Le Matin, les affaires et la polique (1884-1897) », Le Mouvement social, $\mathrm{n}^{\circ}$ 232, juillet-septembre 2010, p. 91-109.

21 Braudel Fernand, Labrousse Ernest (éd.), Histoire économique et sociale de la France, t. 4, vol. 1-2, 1880-1950, Paris, Presses Universitaires de France, Quadrige, 1993, 971 p., p. 138.

22 Ibid., p. 286-287.

23 Charle Christophe, Les élites de la République, op. cit., p. 173-175.

24 Ibid.

25 D'après Fred Kupferman, Maurice Bunau-Varilla s'est arrêté à la classe de quatrième (L'évolution politique du Matin, 1903-1914, 1934-1940, DES d'Histoire, Faculté des Lettres de Paris, 164 p., p. 34). 
aux sphères du pouvoir que grâce à sa fortune opportunément investie dans $L e$ Matin. Au cours de cette trajectoire, qui le fait passer du champ économique au champ journalistique, puis au champ du pouvoir, la conversion de capital est difficile. Celui qu'on surnomme "l'Empereur de la Maison rouge " est aussi riche qu'influent (sa fortune est estimée à plus de 40 millions $^{26}$ ), mais son capital financier et social ne parvient pas à compenser son manque de capital scolaire et culturel. Maurice Bunau-Varilla est ainsi toujours craint à cause de l'influence que lui procure Le Matin, mais reste constamment méprisé et n’est jamais pris au sérieux. Comment le souligne avec malice le député Pierre Baudin en 1905, il est considéré comme un personnage «intelligent, mais un peu fruste ${ }^{27} » \ldots$

\section{Les rapports ambigus avec les élites politiques}

Maurice Bunau-Varilla a la même conception dela presse qu'Eugène Letellier, propriétaire du Journal, qui justifie la fondation de ce quotidien au lendemain du scandale de Panama en affirmant : "Mon journal, c'est un pistolet dans ma poche $^{28}$. "Si l'utilisation de la presse à des fins de chantage est alors largement répandue, les relations avec le monde politique ne peuvent pas être uniquement fondées sur la menace, la crainte et le rapport de force. Le patron du Matin doit notamment développer une sociabilité particulière, faite de services rendus, d'hospitalité et, parfois, de complicité, voire d'intimité. Certains lieux de sociabilité privilégiés sont révélateurs de cette proximité qui dépasse le simple rapport de force. Lopéra, notamment, sert à Bunau-Varilla pour entretenir ses relations. En 1904, il obtient du directeur de l'Opéra, Gailhard, d'avoir à sa disposition la loge de Marinoni (ancien directeur du Petit Journal) après le décès de ce dernier. Dix places situées à l'avant-scène peuvent ainsi être utilisées chaque mercredi par Le Matin, qui a « la possibilité d'être agréableà certains collaborateurs occasionnels pour lesquels une rémunération pécuniaire est impossible ${ }^{29}$. " Bunau-Varilla s'en sert aussi pour offrir des places à divers ministres, comme en 1905 à Fernand Dubief, ministre du Commerce, de l'Industrie et des PTT ${ }^{30}$. Les lieux publics ne remplacent cependant pas l'intimité de l'espace privé : alors qu'Edwards échoue à ouvrir un salon à son hôtel particulier de la rue SaintGeorges (ancien hôtel particulier de Moïse Polydore Millaud, fondateur du Petit

26 La fortune de Maurice Bunau-Varilla est évaluée en 1908 à 40 millions par le sénateur Charles Humbert (Le Journal, $1^{\text {er }}$ juillet 1908) et 50 millions par l'ancien journaliste du Matin François-Ignace Mouthon (Mouthon François-Ignace, Du bluff au chantage. Les grandes campagnes du Matin, Paris, Pauwels, 1908, 196 p., p. VIII).

27 Le Journal, 27 juin 1908.

28 Cité in Garrigues Jean, Les Patrons et la politique. De Schneider à Seillière, Paris, Perrin, 2002, 336 p., p. 53.

29 AN (Archives nationales), 1 AR 8, Conseil d'administration de la société « Le Matin », 6 avril 1904.

30 AN, 18 AR 1, Dubief à Bunau-Varilla, 23 mars et 23 mai 1905. 
Journal), Bunau-Varilla reçoit personnalités, députés, ministres et chefs d'État à son hôtel particulier du 22, avenue du Président Wilson (près du Trocadéro) ou à son château situé à Orsay. Maurice Maillard, secrétaire de la Direction générale des services du Matin puis administrateur de 1925 à 1940, se souvient de l'emploi de temps chargé de son ancien employeur :

Je peux affirmer qu'aux déjeuners d'Orsay ou de l'avenue Wilson Bunau-Varilla a reçu tout le monde politique influent, présidents du Conseil et ministres en tête. Il n'a pas déjeuné hors de chez lui plus de trois ou quatre fois dans sa vie. [...] La semaine se déroulait ainsi :

Le lundi, réception à Paris : quelques grands patrons du corps médical, « les plus grands ».

Le mardi, réception à Orsay : hommes politiques, ambassadeurs ou sommités étrangères de passage en France ${ }^{31}$.

Le mercredi, réception à Paris : hommes politiques en fonction, dont le temps était plus compté.

Le jeudi, réception à Orsay : même déjeuner que le mardi.

Le vendredi, réception à Paris : même déjeuner que le mercredi.

Le samedi, réception à Orsay : en général quelques amis politiques qui passaient la journée avec lui ${ }^{32}$.

Cependant, cette proximité est généralement factice et reste dépendante de l'influence dont le patron du Matin dispose, qui fait de lui un personnage dangereux qu'il faut savoir ménager. Certes, Aristide Briand devient, à partir de 1917, un proche de Bunau-Varilla, mais il est difficile de savoir quelle est la part d'intimité réelle et d'opportunisme politique de la part de celui qui est Président du Conseil et ministre des Affaires Étrangères à plusieurs reprises, comme le suggère Georges Suarez :

De ces longs contacts étaient née entre les deux hommes, non pas seulement de l'amitié, mais une confiance si parfaite qu'elle n'excluait de leurs entretiens aucun domaine. Ils avaient les mêmes pensées sur bien des choses, et les souvenirs que Briand évoquaient sur son passé, son enfance, sa dure jeunesse rendaient plus perceptibles encore, à son perspicace interlocuteur, certaines positions psychologiques. Chez Bunau-Varilla, il rencontrait tous les chefs et les hommes publics français et alliés que les événements mettaient en vedette. C'était pour lui une source inépuisable d'informations avec quoi il pouvait discerner objectivement la politique possible de celles qui ne l'étaient pas ${ }^{33}$.

31 En 1925, Bunau-Varilla reçoit notamment à Orsay le roi d'Angleterre George V, qui le remercie par écrit, le 5 septembre : « Je ne veux pas quitter Paris sans vous exprimer de la part de la Reine et de la mienne, nos sentiments affectueux et nos remerciements pour l'attention dont nous avons été l'objet dans votre merveilleux pays. La journée que nous avons passée à Orsay, dans votre château, sera pour nous un souvenir charmant. Je vous envoie de notre part la photo de la Reine et la mienne. Je désire que notre image vous reste fidèle comme l'amitié que nous vous portons. " (cité in Kupferman Fred, L'évolution politique du Matin, op. cit., p. 122)

32 Témoignage écrit de Maurice Maillard, 1958, cité in Kupferman Fred, L'évolution politique du Matin, op. cit., p. 33.

33 Suarez Georges, Briand: sa vie, son œuvre, avec son journal et de nombreux documents inédits, t. 4, Le pilote dans la tourmente : 1916-1918, Paris, Plon, 1940, 396 p., p. 321. 
Raymond Poincaré, avocat du Matin à partir de 1894 et jusque dans les années 1900, est, lui aussi, très proche de Bunau-Varilla pendant de longues années, au point de devoir subir sa correspondance déconcertante ${ }^{34}$. Mais, en 1928, l'ancien Président de la République n'hésite pas à s'en éloigner, au moment où Le Matin a déjà fortement décliné35. L'Empereur de la Maison rouge ne semble ainsi disposer que d'une influence directement liée à l'audience de son journal ; il n'est pas respecté, mais craint. Se rêvant en éminence grise du gouvernement ou en conseiller discret et écouté, le patron du Matin, dont la mégalomanie n'a d'égale que la vénalité, se distingue le plus souvent par son ridicule. Et s'il fait effectivement trembler députés, ministres et chefs d'État au moment où Le Matin connait son « âge d'or » (du milieu des années 1900 jusqu’à la fin de la Première Guerre mondiale), ses habitudes de petit tyran provoquent de plus en plus le rire au fur et à mesure que son journal s'affaiblit. Dans la seconde moitié des années 1920, le ministère des Affaires Étrangères allemand réussit ainsi à acheter la bienveillance du Matin en promettant à Bunau-Varilla de diffuser en Allemagne la solution qu'il considère alors comme une panacée et qu'il produit lui-même en France : le Synthol ${ }^{36}$. Si cette opération de subvention indirecte révèle l'influence dont Le Matin dispose encore à l'époque malgré les difficultés auxquelles il doit faire face, elle est aussi très représentative de la manière dont le patron de la Maison rouge est considéré à l'étranger. Alors que BunauVarilla terrorisait le roi des Belges Léopold II en 1904 (lors d'une campagne de chantage qui s'avéra très fructueuse) ou le gouvernement du Tsar avant la Première Guerre mondiale (à l'occasion du lancement des emprunts russes), la Wilhelmstrasse l'instrumentalise tout en s'en moquant, comme le révèle l'anecdote rapportée par Pierre Lazareff:

Otto Abetz raconte plus tard en éclatant de rire comment son collègue, le représentant des industries chimiques allemandes, avait, pour flatter l'orgueil du vieux Maurice Bunau-Varilla, déclaré en signant le contrat : "Quelle que soit la science de nos savants, Monsieur, ils ont été stupéfaits par le Synthol. Ils ont réussi à en faire l'analyse, ils sont incapables d'en faire la synthèse ». Le potentat du Matin l'aurait embrassée ${ }^{37}$ !

\section{Conclusion}

Le Matin est un cas trop particulier pour que les conclusions qui le concernent puissent être étendues à l'ensemble de la presse, mais son histoire est révélatrice des rapports qu'entretiennent les journaux avec les élites politiques

34 À titre d'exemple, voir la lettre de Maurice Bunau-Varilla à Raymond Poincaré du 5 novembre 1928, in Pinsolle Dominique, Le Matin (1884-1944), op. cit., p. 310-311.

35 Ibid., p. 215-216.

36 Bariéty Jacques, "Le Matin et l'Allemagne à l'époque de la République de Weimar », in Presse et politique, Actes du colloque de Nanterre, mars 1973, Les Cahiers du CEREP, $\mathrm{n}^{\circ} 1$.

37 Lazareff Pierre, De Munich à Vichy, New York, Brentano, 1944, 355 p., p. 78. 
et économiques. L'émergence d'une presse dite " d'information " s'accompagne incontestablement de la montée en puissance d'une nouvelle catégorie de travailleurs intellectuels dont l'élite dispose d'une influence indéniable. Le pouvoir de cette élite journalistique reste cependant ambigu, dans la mesure où il ne remet pas fondamentalement en cause celui des élites traditionnelles, qu'elles soient politiques ou économiques. Si ces dernières sont désormais obligées de composer avec la presse, et notamment les journaux de chantage comme Le Matin, leur position n'est pas véritablement menacée. Les patrons de presse, qui peuvent, comme Maurice Bunau-Varilla, accéder au champ du pouvoir sans disposer au départ de toutes les formes de capitaux nécessaires, sont, certes, très puissants dans certaines circonstances, mais restent dépendants du succès de leur journal, qui constitue la base de leur influence. Par ailleurs, leur marge de manœuvre demeure limitée, dans la mesure où, malgré le sentiment de toute-puissance que peut procurer la possession d'un grand quotidien, les élites politiques et économiques conservent des moyens de pression dont nul ne peut totalement s'affranchir. Soucieux d'assurer à son journal les «scoops » les plus retentissants, un patron de presse devra s'efforcer de rester proche du pouvoir pour être au plus près des sources d'information, comme le souligne Michael B. Palmer, pour qui «malgré les apparences du contraire, le quotidien d'information est du côté des puissants ":

En fait, se targuant d'en savoir aussi long sur l'actualité que ses acteurs, les quotidiens à grand tirage se penchent, le plus souvent, du côté des initiés, dans leur manière de présenter certaines informations. Ils sont d'autant plus prévenants que leurs interlocuteurs disposent, en tant que sources, de moyens puissants à leur égard. Ils le sont moins, dans les récits criminels et autres faits divers, où Monsieur Tout-le-Monde est le héros passager de leur histoire ${ }^{38}$.

En tant qu'hommes d'affaires, des patrons de presse comme Alfred Edwards ou Maurice Bunau-Varilla ont également besoin de rester proches du pouvoir pour bénéficier de protections à chaque fois que leurs affaires sont menacées. Quant aux puissances d'argent, ils en sont tout autant dépendants lorsque les caisses de leur journal ont besoin d'être renflouées... La multiplicité des liens qui unissent Le Matin aux élites politiques et économiques remet donc radicalement en cause la notion de "contre-pouvoir " ou de "quatrième pouvoir " en ce qui concerne la presse : dans le cas que nous avons étudié, la presse s'avère être davantage un auxiliaire du pouvoir.

\section{Dominique Pinsolle}

Université Bordeaux Montaigne Dominique.Pinsolle@u-bordeaux-montaigne.fr

38 Palmer Michael B., Des petits journaux aux grandes agences, op. cit., p. 17-18. 


\section{Résumé}

Les journaux, dont le nombre croît fortement au cours du XIX ${ }^{\mathrm{e}}$ siècle, sont généralement vus comme un "Quatrième Pouvoir ", ou un " contre-pouvoir ". Dans la mesure où ils informent la population, ils sont censés être un élément essentiel du système démocratique. Mais les liens entre la presse (qui est presque totalement libre en France après 1881) et les élites politiques et économiques sont ambigus. Le Matin, par exemple, un des quotidiens français les plus populaires durant la première moitié du XX $\mathrm{XX}^{\mathrm{e}}$ siècle, prétend toujours être le porte-parole du peuple contre les élites corrompues. Il organise ainsi de grands événements populaires ou de grandes campagnes retentissantes durant lesquels il attaque des élites politiques et économiques défaillantes au nom de la nation. Mais en réalité, ce journal est très proche de certains hommes d'affaires et de certains hommes politiques, tout en étant également lui-même corrompu à de nombreuses reprises.

\section{Mots-clés}

Presse, élites, quatrième pouvoir, milieux d'affaires, pouvoir politique.

\section{Abstract}

The newspapers, whose number grows dramatically during the XIXth century, are generally seen as a "Fourth Power" or a "counter-power". As they inform people, they are supposed to be an essential part of the democratic system. But the links between the press (which is almost totally free after 1881 in France) and the business and the political elites are ambiguous. Le Matin, for example, one of the most popular French dailies during the first part of the XXth century, always claims to be the voice of the people against the corrupt French elites. It organizes big popular events or campaigns during which it attacks failing business and political elites in the name of the nation. But in reality, this newspaper is very close to certain businessmen and politicians, and is itself many times corrupt.

\section{Keywords}

Press, elites, "Fourth power", business, political power. 